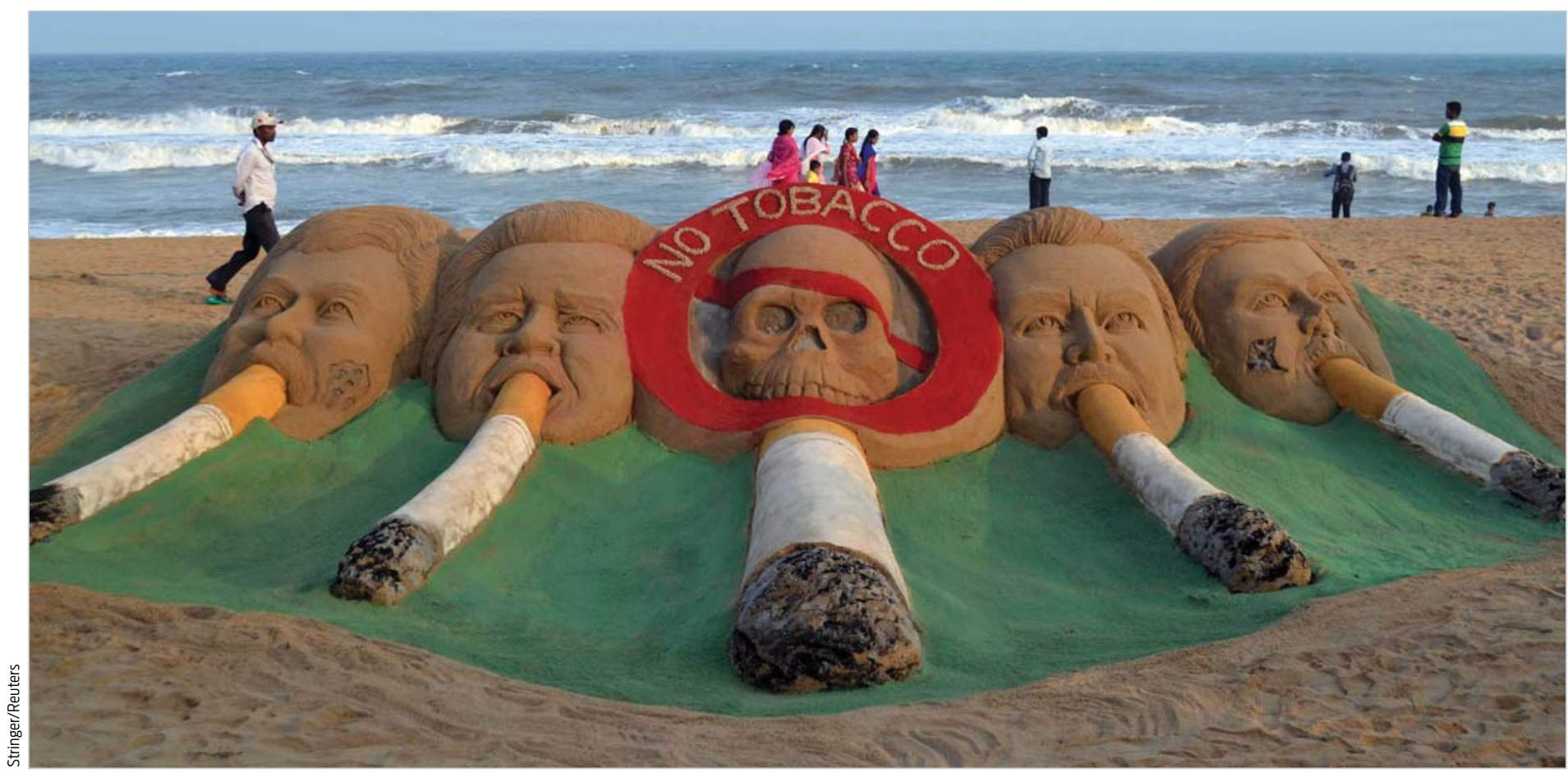

Visitors walk next to a sand sculpture made by Indian sand artist Sudarshan Pattnaik on World No Tobacco Day, 31 May, at a beach at Puri in the eastern Indian state of Odisha.

\title{
The shape of things to come: visions for the future of Aboriginal and Torres Strait Islander health research
}

In 2013, the Lowitja Institute embarked on a project using futures thinking to consider how research might best contribute to Aboriginal and Torres Strait Islander health and wellbeing in the year 2030. Futures thinking - a growing discipline in research and management - is not about predicting the future, but rather about identifying a number of possible and plausible futures. It differs from traditional research methods in that it draws on deeper intuitive insights as well as hard evidence.

Workshops were held nationally to consider possible scenarios based on two divergent futures: an inclusive, vibrant Australia in which Aboriginal and Torres Strait Islander cultures are valued and embraced as central to the Australian identity; and an Australia in which economic and spiritual poverty drive a rejection of diversity and increase the divide between rich and poor.

The response from our consultations was not simply a list of research topics. Rather, participants articulated a strong and widely shared desire for a profoundly different system of research - a system in which research and practice are closely interwoven and which enables greater integration of health services, policy and research. Such a system would be responsive to changing research demands, but also to changing social, economic, technological and knowledge landscapes. This builds on work and methods developed by the Lowitja Institute and its predecessor organisations over the past 20 years.

Consultations also identified an urgent need to address the social

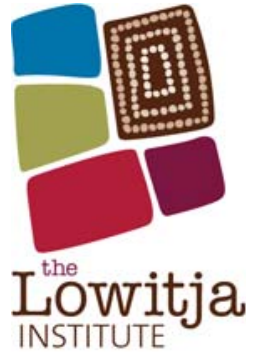

Mary Guthrie

General Manager, Policy and Communications Lowitja Institute

doi: 10.5694/mjal4.00666 determinants of Aboriginal and Torres Strait Islander health, along with a growing sense that the health and health research sectors need to play a facilitating role, inviting other sectors - such as early childhood, education, justice and local government - to collaborate and maximise the impact of their collective efforts to bring about change.

Finally, the project identified that the Aboriginal and Torres Strait Islander health and health research sectors have played a pioneering role in the reform of research in Australia. In its role as an enabler of health research solutions, the Lowitja Institute will work with its partners to respond to this vision in our current and future work.

The report is available at https:// www.lowitja.org.au/lowitja-publishing. 


\section{Damning report on Nauru health care}

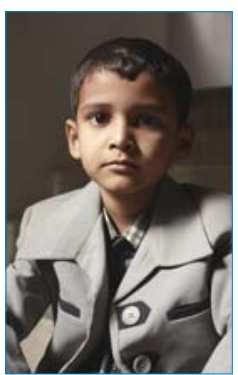

The Guardian has published a report by five independent clinical experts on the state of health care offered to asylum-seeker families, children, babies and pregnant women inside the Nauru detention centre. The long list of health risks includes crowded, hot conditions with little physical activity; lack of mental health screening or support despite 102 incidents of self-harm in 14 months; lack of health checks for children; gaps in immunisation programs; lack of paediatric expertise; lack of child protection capacity; and poor maternal services. There have also been reports of dengue fever and groundwater contamination.

\section{Unintended consequences and the $\mathrm{CIA}$}

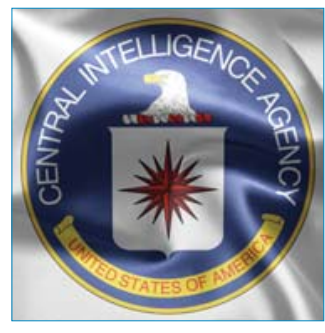

In the hunt for the late Osama bin Laden in Pakistan, Central Intelligence Agency officers used polio vaccination programs to obtain DNA evidence to confirm the presence of the bin Laden family, The Lancet reports. As a result, legitimate health care workers have been targets for violence from militants believing them to be United States spies. Organisations such as the United Nations have been forced to suspend polio vaccination programs. The World Health Organization has declared that polio has re-emerged as a public health emergency in Pakistan. The White House has now banned the use of vaccination programs as cover for espionage.

\section{Google Glass making it mainstream}

The wearable computer, Google Glass, is finding a place in medicine. The New York Times reports that an increasing number of surgeons in the US are testing Glass as a means of broadcasting and recording surgery from their point of view. There is also the possibility of laparoscopic images being projected in front of the surgeon rather than from a

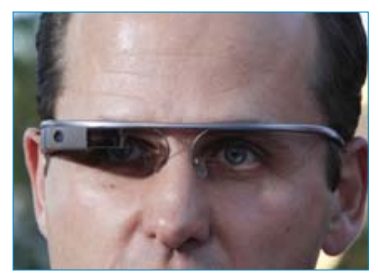

screen across the operating theatre. The downsides? Distraction, privacy issues and tunnel vision, according to detractors. Meanwhile the University of California-Irvine claims to be the first to introduce Glass into their School of Medicine's classrooms.

\section{Payout on US dabigatran lawsuits}

German pharmaceutical company Boehringer Ingelheim will pay US\$650 million to settle some 4000 lawsuits in the US connected with its novel oral anticoagulant dabigatran, an average of US\$162 500 per claimant, Drugwatch reports. According to the US Food and Drug Administration, more than 540 patients died in 2011 following the use of dabigatran, while thousands more suffered serious side effects. The lawsuits argued that dabigatran can "cause bleeding events that cannot be controlled and are sometimes fatal". In 2012, sales of dabigatran hit US $\$ 1.5$ billion. Two other novel oral anticoagulants have since been approved: Johnson \& Johnson's rivaroxaban and Pfizer and Bristol-Myers Squibb's apixaban.

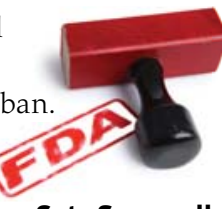

Cate Swannell doi: 10.5694/mjal4.n0616

\section{MJA InSight poll Totalrespondents:269}

Has there been an
the medical and g
communities to th
introduction of a
copayment for GP
and diagnostic
services?
MJA InSight

Take part in next week's poll on: www.mja.com.au/insight

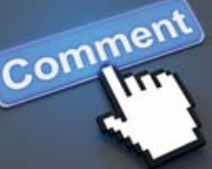

Suggested topic:

Is it time Australia joined

Austria, Belgium, Canada,

Czech Republic, Finland,

Israel, the Netherlands, Spain, the UK

and some US states in legalising cannabis

for use by the terminally ill?

Email your comment to: comments@mja.com.au

\section{Are not these biscuits Aussie-owned?}

I refer to your "Highlights from our archives" in the Journal of 2 June, page 555 . The article contains a well remembered Arnott's advertisement for its well known Scotch Fingers. There is something very sad about this - though this product is still available, the company itself, once an Australian icon, is now overseas owned!

Dr Kevin B Orr Retired surgeon, NSW.

\section{The truth about euthanasia}

With Dr Philip Nitschke publicly supporting assisted suicide for people aged over 50 years who are healthy but "tired of living", it is no wonder that politicians continually reject euthanasia bills. Dr Nitschke even calls his Adelaide clinic an "exit clinic" not a euthanasia clinic. He is currently running workshops around the country selling nitrogen gas suicide kits to people who are not terminally ill. There have been many complaints made against Dr Nitschke, but the Australian Health Practitioner Regulation Agency refuses to release the findings of its investigations. It is time the public were told the truth about euthanasia.

Dr John Hayes Physician, WA.

\section{Can you beat this!}

Tidying up my Mac's desktop, I find that I have had 50 contributions published in the MJA since my first in 1965.

Does the MJA have the equivalent of the Sydney Morning Herald's Column8 PhD?

Dr Peter Arnold

Retired general practitioner, NSW.

Email us a brief comment (no more than 100 words) on any healthrelated subject. Email your comments to: comments@mja.com.au When sending your comment, please include your full name, discipline and state of residence, and disclose any relevant information or affiliations that may affect interpretation of your comments.

Visit: https://www.mja.com.au/journal/mja-instructions-authorstypes-articles-published-mja\#Comments 